\title{
Identification of a Novel Secretory Gland Producing C-type Lectin in the Flesh Fly (Sarcophaga peregrina), and its Characterization
}

\author{
Mami YAMAMOTO-KIHARA ${ }^{1 *, 2}$, Fumiko YUKUHIRO ${ }^{1}$, Hiroshi YASUE ${ }^{3}$, \\ Eiji KOTANI ${ }^{2,4}$ and Hajime MORI ${ }^{2,4}$
}

\author{
${ }^{1}$ Division of Insect Science, National Institute of Agrobiological Sciences \\ (Tsukuba, Ibaraki 305-8634, Japan) \\ ${ }^{2}$ Graduate School of Science and Technology, Kyoto Institute of Technology \\ (Sakyo-ku, Kyoto 606-8585, Japan) \\ ${ }^{3}$ Agrogenomics Research Center, National Institute of Agrobiological Sciences \\ (Tsukuba, Ibaraki 305-8602, Japan) \\ ${ }^{4}$ Insect Biomedical Research Center, Kyoto Institute of Technology \\ (Sakyo-ku, Kyoto 606-8585, Japan)
}

\begin{abstract}
C-type lectin-producing tissue, designated as CLPT, constitutes mouthparts of the flesh fly (Sarcophaga peregrina) and produces a C-type lectin, CLEM 36, which is found to be secreted into saliva. In the present study, CLPT was investigated by immunohistochemical method using a transmission electron microscope (TEM) and scanning electron microscope (SEM), in order to clarify the secretion pathway of CLEM 36. CLPT was found to consist of cells having a microvillus. Each CLPT cell was connected to a fine duct. The fine ducts were bundled together and connected to the food canal. CLEM 36 was densely detected in the microvilli of CLPT cells, and also detected in cytoplasm, and ducts of CLPT. CLPT was found not to be connected to the salivary glands. Since little expression of CLEM genes including CLEM 36 was detected in the thorax and abdomen including the salivary glands in our previous study, when taken together, our current findings suggest that CLPT is a novel secretory gland which is independent of salivary glands, and has its own role in the intake of foods.
\end{abstract}

Discipline: Insect pest

Additional key words: azan staining, toluidine blue staining, whole mount immunostaining

\section{Introduction}

Flesh flies in the genus Sarcophaga (Diptera: Sarcophagidae) have been important models in insect physiology and biochemistry, particularly in the fields of endocrinology (Fraenkel et al. 1972, Verleyen et al. 2004), diapauses (Denlinger 2002, Hahn \& Denlinger 2007) and cold hardiness (Lee et al. 1987, Rinehart et al. 2007). Most flesh flies breed on carcasses and excrements, and survive in an environment full of parasites, bacteria and other infectious organisms. An effective defense system is considered to exist in these species, and therefore, flesh flies are a useful model for studying innate immunity (Natori et al. 1999, Tanji et al. 2008).

Lectins are widely distributed in animals, plants, and bacteria, and have various biological activities such as those working in defense systems against pathogens.
Animal lectins have been generally classified into Ctype, S-type, P-type, and non-classified based on their carbohydrate recognition. Especially in insects, secretory C-type lectins are assumed to play roles in mediating cellular and cell-substrate interaction during developmental processes and in the immune system (Kawaguchi et al. 1991, Natori 2001). Two lectins, Sarcophaga lectin and granulocytin, isolated from larvae of the flesh fly S. peregrina, are surmised to be involved in innate immunity (Takahashi et al. 1985, Fujita et al. 1998). Sarcophaga lectin is reported to be synthesized in fat bodies in response to bodily injury of larvae, and secreted into their hemolymph; and granulocytin, to be continuously synthesized in the hemocytes of naive larvae, and eventually secreted into hemolymph.

We have recently identified two novel C-type lectins in mouthparts of S. peregrina, CLEM 20 and 36 
(Yamamoto-Kihara \& Kotani 2004, Yamamoto-Kihara et al. 2011). CLEM 20 and 36 were characterized as being highly and continuously transcribed from the second day after eclosion only in one tissue, which was designated as C-type lectin-producing tissue (CLPT), located at the entrance to the food canal between the labellum and the haustellum. CLEM 20 and 36 are secreted into saliva, suggesting that they have physiological roles different from Sarcophaga lectin and granulocytin. We also found CLEM 36 protein in CLPT cells using immunostaining of sections (Yamamoto-Kihara et al. 2011). However, the existence of CLEM 36 in the cells would not be decisive evidence yet to suggest that CLPT is a secretory organ.

In the present study, to obtain a clue for elucidating the roles of CLEM 20 and 36 in the adult flesh fly, we have morphologically investigated CLPT and performed comprehensive analysis for the existence of CLEM 36 in CLPT using whole mount and TEM-based immunostaining. We then identified it as a novel secretory gland producing C-type lectin in the flesh fly.

\section{Materials and methods}

\section{Fly samples}

Flesh flies (Sarcophaga peregrina) were maintained at $25^{\circ} \mathrm{C}$ and reared on $3 \%$ sucrose and fresh water. Pork liver was also provided for depositing larvae. Larvae were reared on pork liver at $20^{\circ} \mathrm{C}$, and pupae were maintained at $20^{\circ} \mathrm{C}$. For immunostaining and histological analyses excluding azan staining, flies were used 3 to 5 days after eclosion. For azan staining, tips of mouthparts were excised just before eclosion.

\section{Azan staining}

Tissue samples were fixed at $4^{\circ} \mathrm{C}$ overnight by immersion in $4 \%$ paraformaldehyde in $0.1 \mathrm{~mol} / \mathrm{L}$ phosphate buffer (PB) (pH 7.4), and washed in $0.1 \mathrm{~mol} / \mathrm{L} \mathrm{PB} \mathrm{(pH}$ 7.4). They were then dehydrated in a graded ethanol series and embedded via xylene intermedium into paraffin (Wako Pure Chemical, Osaka, Japan). Paraffin sections 4 $\mu \mathrm{m}$ thick were stained according to Mallory-Heidenhain (Bancroft \& Gamble 2001), and observed by light microscope (DMLB; Leica Microsystems, Buffalo Grove, IL, USA).

\section{Toluidine blue staining}

Tips of mouthparts were fixed as described above, and subjected to dehydration in a graded ethanol series and to infiltration via propylene oxide intermedium with EPON 812 resin (TAAB Laboratories Equipment Ltd., Berkshire, UK) (overnight in 50\% EPON 812 in propylene oxide, $3 \mathrm{~h}$ in $75 \%$ EPON 812 in propylene oxide, $2 \mathrm{~h}$ in $100 \%$ EPON 812 and $24 \mathrm{~h}$ in fresh $100 \%$ EPON 812) at $4{ }^{\circ} \mathrm{C}$, followed by polymerization of the resin at $60^{\circ} \mathrm{C}$ overnight. Sections with a thickness of $1 \mu \mathrm{m}$ were stained with toluidine blue, and inspected by light microscope (DMLB).

\section{Whole mount immunostaining analysis}

Tips of mouthparts were fixed as described above and digested with Protease $\mathrm{K}$ according to the procedure described previously (Yamamoto-Kihara \& Kotani 2004), followed by washing twice with phosphate buffer saline (PBS) containing glycine and with PBS containing $0.3 \%$ Triton X-100 (PBST). Blocking was performed in blocking buffer (PBST containing 3\% BSA) for $2 \mathrm{~h}$. Samples were incubated with the anti-CLEM 36 antiserum (1:800) (Yamamoto-Kihara \& Kotani 2004) or the preimmune serum $(1: 800)$ in blocking buffer at $4^{\circ} \mathrm{C}$ for more than $16 \mathrm{~h}$. CLEM 36 was recognized and detected using a VECTASTAIN Universal Elite ABC Kit (Vector Laboratories, Burlingame, CA, USA) and VectaStain VIP Substrate Kit for Peroxidase (Vector Laboratories), according to the manufacturer's instructions.

\section{Scanning electron microscopy}

Tips of mouthparts were excised, and the cuticles were removed from the tips. The samples thus obtained were then fixed with $4 \%$ paraformaldehyde in PBS at $4^{\circ} \mathrm{C}$ overnight, followed by washing with PBS. For SEM analysis, two types of specimens were prepared from the fixed samples; one was the outer matrix of the mouthpart tips treated with protease $\mathrm{K}$ at a concentration of $1 \mu \mathrm{g} / \mathrm{ml}$ for $10 \mathrm{~min}$ at $25^{\circ} \mathrm{C}$; the other was the sagittally-dissected mouthpart tips without protease treatment. The protease K-treated samples were washed in PBS containing glycine and refixed with $4 \%$ paraformaldehyde in PBS for 2 $\mathrm{h}$ at $25^{\circ} \mathrm{C}$, followed by washing with PBS. The resulting samples were dehydrated with ethanol series, and dried via isopentyl acetate by the critical point method using $\mathrm{CO}_{2}$ (Anderson, 1951). The dried samples were coated with gold, followed by examination with SEM (6301 F; JEOL, Tokyo, Japan) at an accelerating voltage of $5 \mathrm{kV}$.

\section{Transmission electron microscopy}

Tips of mouthparts were excised and frozen immediately in liquid nitrogen at high pressure using a Leica EM PACT high-pressure freezer (Leica Microsystems). Freeze substitution of the frozen specimens was conducted using an AFS system (Leica Microsystems) beginning at $-90^{\circ} \mathrm{C}$ in $1 \% \mathrm{wt} / \mathrm{vol}$ osmium tetroxide in acetone, and ending in several changes of EPON 812 resin at $20^{\circ} \mathrm{C}$ through pure acetone at $-50^{\circ} \mathrm{C}$. The resin containing specimens was polymerized at $60^{\circ} \mathrm{C}$ for $24 \mathrm{~h}$. Ultra-thin 
sections $(60 \mathrm{~nm})$ of the samples were obtained using a Leica Ultracut R (Leica Microsystems), and were stained with uranyl acetate and lead citrate. Samples were studied by TEM (JEM-1010, JEOL) at an accelerating voltage of $80 \mathrm{kV}$.

\section{TEM-based immunostaining}

Tips of mouthparts were removed, cut in half, and fixed with $1 \%$ paraformaldehyde and $0.25 \%$ glutaraldehyde in $0.1 \mathrm{~mol} / \mathrm{L} \mathrm{PB}(\mathrm{pH} 7.4)$ at $4^{\circ} \mathrm{C}$ overnight, followed by washing with $0.1 \mathrm{~mol} / \mathrm{L} \mathrm{PB}(\mathrm{pH} 7.4)$. Samples were dehydrated in a graded ethanol series and infiltrated with Lowicryl K4M resin (Chemische Werke Lowi GmbH \& Co., Waldkraiburg, Germany) overnight in 50\% K4M in ethanol, $3 \mathrm{~h}$ in $75 \% \mathrm{~K} 4 \mathrm{M}$ in ethanol, $2 \mathrm{~h}$ and $24 \mathrm{~h}$ in $100 \%$ $\mathrm{K} 4 \mathrm{M}$, at $4^{\circ} \mathrm{C}$. After transferring the samples to gelatin capsules with fresh $\mathrm{K} 4 \mathrm{M}$ resin, they were polymerized using ultraviolet light at $4^{\circ} \mathrm{C}$ for $65 \mathrm{~h}$. Ultra-thin sections $(60 \mathrm{~nm})$ of the CLPT on nickel grids covered with
$2 \%$ collodion solution in isoamyl acetate were treated with antibody against anti-CLEM 36 and then with 20 nm-gold labeled goat-rabbit $\operatorname{IgG}(\mathrm{H}+\mathrm{L})$, according to the manufacturer's instructions (BBInternational, Dundee, UK), and examined with TEM.

\section{Results}

Under SEM observation, a pair of CLPT was localized at the entrance to the food canal between the labellum and haustellum (Fig. 1iii), consistent with previous observations (Yamamoto-Kihara \& Kotani 2004). Furthermore, SEM examination demonstrated that most CLPT cells were of a round shape with an approximate diameter of $25 \mu \mathrm{m}$ (Fig. 1v). Each CLPT cell was connected to a fine duct, forming a botryoid shape (Figs. liv-vi). The fine duct showed a stripe structure on its surface, which was different from the cell membrane of the CLPT (Figs. 1vii-viii). The ducts were bundled and connected with the entrance to the food canal (Fig. 1iv). The

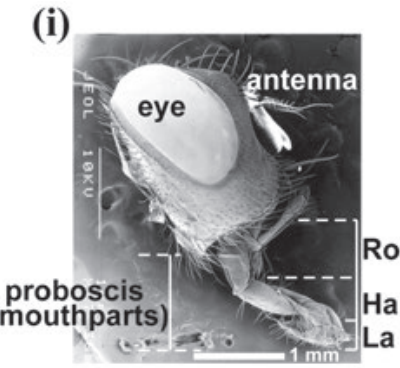

(ii)

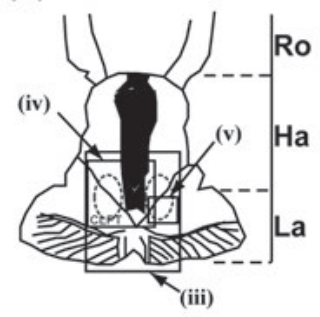

(v)
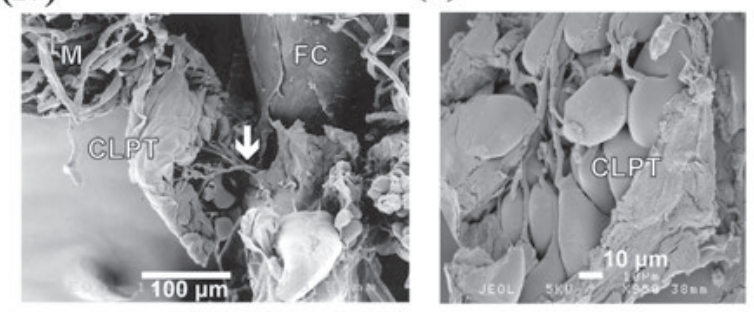

(vi)

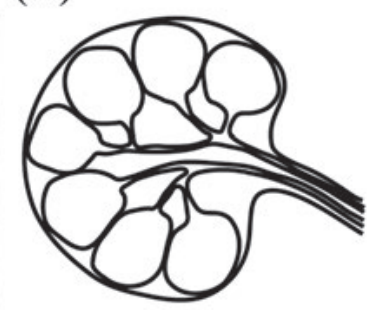

(iii)

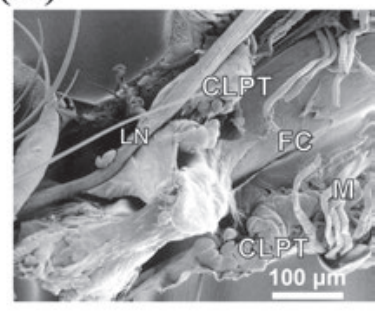

(vii)

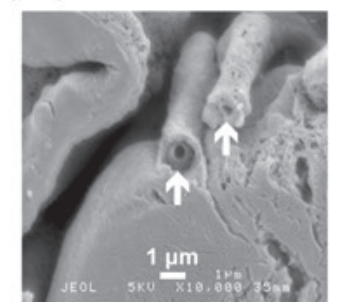

(viii)

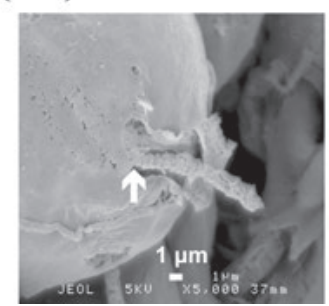

Fig. 1. SEM images of flesh fly head region

(i) Represents the head of the flesh fly; and (ii) is a schematic drawing of the proboscis shown in (i). (iii) - (v) Images correspond to the respective areas in the drawing (ii). (vii) And (viii) present cells and ducts of CLPT, which are shown in (iii) - (v). (vi) Represents a schematic drawing of CLPT seen in (iv) - (v). The white arrow in (iv) indicates bundled ducts connecting to the food canal; white arrows in (vii), ducts; and the white arrow in (viii), protraction site of duct from a CLPT cell. Ro represents rostrum; Ha, haustellum; La, labellum; FC, food canal; M, muscle; and LN, labial nerve. 
(i)

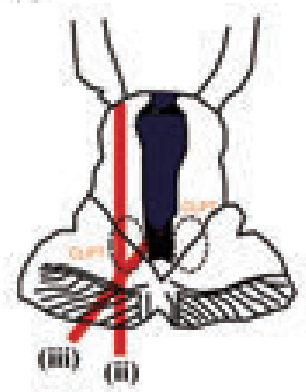

(ii)

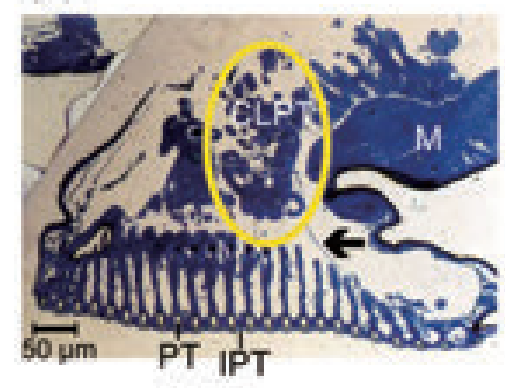

(iii)

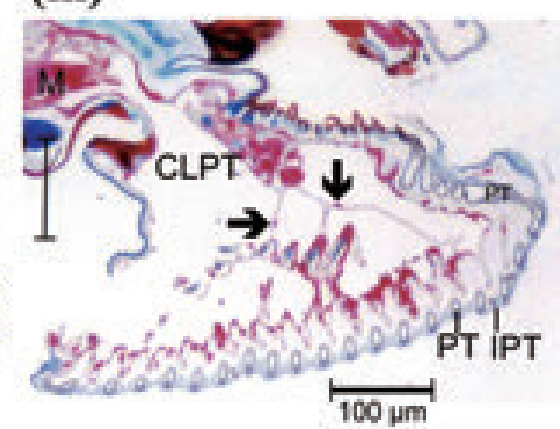

Fig. 2. Photographs of toluidine blue and azan staining of the tip of mouthparts

(i) Schematic drawing of the proboscis. Red lines indicate the dissection positions for toluidine blue staining (ii), and azan staining (iii). CLPT is shown as a yellow ellipse in (ii). Black arrows indicate the cord linking CLPT with the pseudotrachea and interpseudotrachea. M indicates muscle; PT, pseudotrachea; and IPT, interpseudotrachea.

(i)

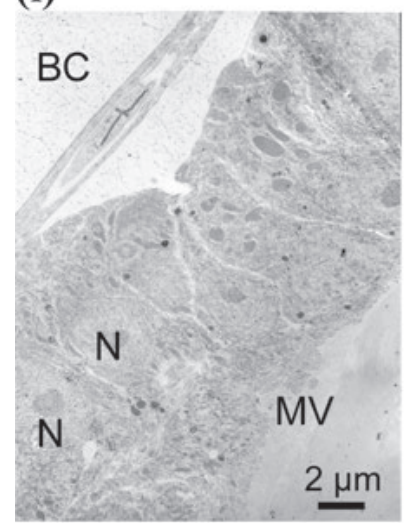

(ii)

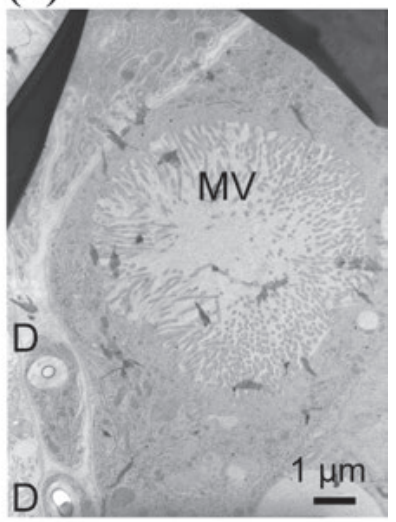

Fig. 3. TEM micrographs of CLPT

Axial (i) and longitudinal sections (ii) of CLPT are shown. N indicates nucleus; MV, microvillus; BC, body cavity; and D, ducts of CLPT.

\section{(i)}

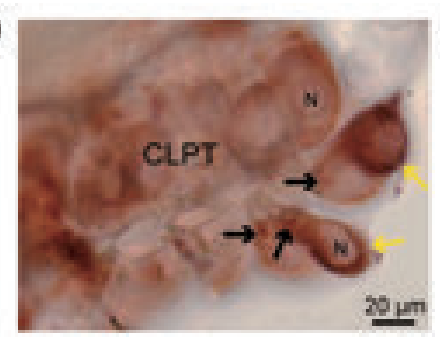

(iii)

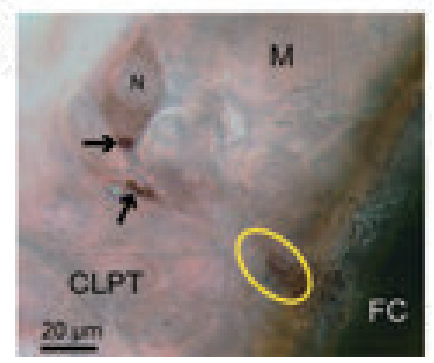

(ii)

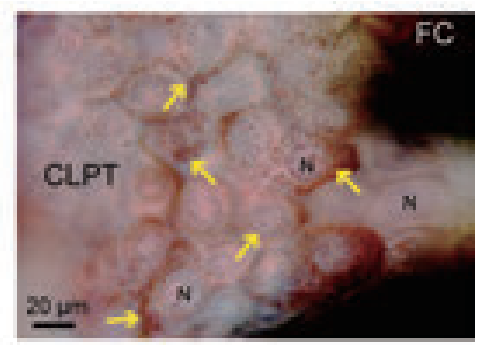

(iv)

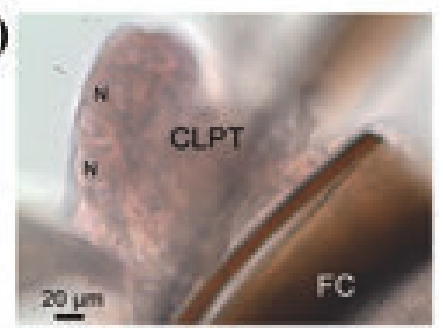

Fig. 4. Whole mount immunostaining using antibody against CLEM 36

(i) - (iii) Whole mount immunostaining using antibody against CLEM 36. CLEM 36 is shown by red staining. Yellow arrows indicate small red spots in cytoplasm, shown in (i) and (ii). Black arrows in (i) and (iii) indicate positions where CLEM 36 is densely localized in cytoplasm. Yellow ellipse in (iii) indicates CLEM 36 in the duct. (iv) Whole mount immunostaining using a preimmune serum. M shows muscle; FC, food canal; and N, nucleus. 
(iii)

(i)

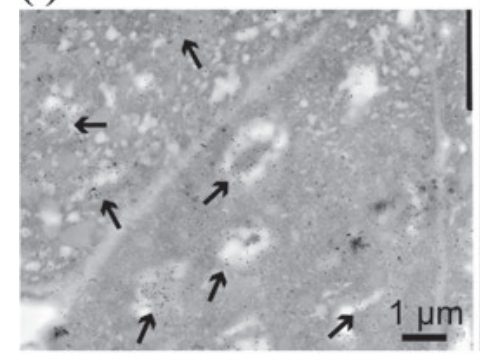

(ii)
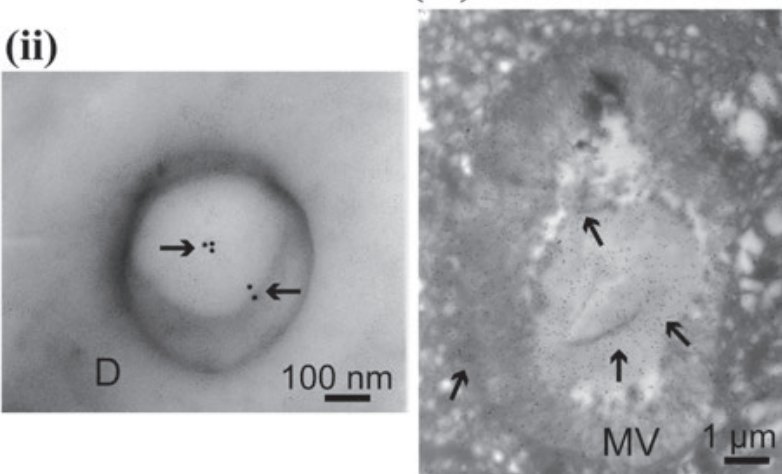

Fig. 5. TEM-based immunostaining using the anti- CLEM 36 serum

Images in this figure are obtained from longitudinal sections of CLPT. Black arrows indicate representative positive signals for CLEM 36. CLEM 36 is localized in cytoplasm of CLPT cells (i), duct (ii) and microvillus (iii). D shows a duct of CLPT and MV, microvillus.

labial nerve was not connected to the CLPT (Fig. 1iii).

The cells located inside of labellar lobes, including the pseudotrachea and interpseudotrachea, were found to form a comb-like alignment (Fig. 2ii). Labellar lobes were found to be connected to CLPT through a cord-like structure containing azan-positive materials (Fig. 2). TEM examination revealed that the cells in CLPT were arranged so as to present their microvilli in an inward direction (Fig. 3).

The analysis using whole mount and TEM-based immunostaining revealed CLEM 36 to be found in the cytoplasmic vesicular inclusions of CLPT cells (Figs. 4iiii, Fig. 5i), and in the content of ducts (Fig. 4iii, Fig. 5ii). In addition, CLEM 36 was found to be heavily concentrated in microvilli (Fig. 5iii).

\section{Discussion}

In the present study, we investigated CLPT by morphological and immunohistochemical methods. The cellular and histological organization of CLPT indicates that this organ has secretory functions. Histochemical distributions of CLEM 36 in CLPT suggest that this lectin is produced in CLPT cells and secreted into the food canal through the duct. We conclude that CLPT is a novel secretory gland in $S$. peregrina which produces CLEM 36. On the other hands, CLEM 20 was not detected in CLPT by TEM-based immunostaining in spite of the fact that CLEM 20 was indicated to be produced in CLPT (Yamamoto-Kihara et al. 2011). It would be due to the low amount of CLEM 20 in this tissue.

The salivary glands of the adult flesh fly were found to mostly consist of thin tubes, which are in the abdomen on either side of the adult gut (our unpublished data). The two tubes are joined in the anterior region of the thorax to form a common duct leading with an opening in the hypopharynx (our unpublished data); these observations are consistent with those of adult Calliphora (Oschman \& Berridge 1970). The thorax and abdomen including the salivary glands were subjected to an examination in the previous study (Yamamoto-Kihara \& Kotani 2004, Yamamoto-Kihara et al. 2011) to see whether CLEM 20 and 36 expressed in the tissues, and little expression of the genes, if any, was detected. These findings taken together demonstrate that CLPT is independent from salivary glands and has its own role in the intake of foods.

CLEM 20 and 36 were shown to be members of C-type lectins (Yamamoto-Kihara \& Kotani 2004, Yamamoto-Kihara et al. 2011), a group demonstrated to be involved in the self-defense system against pathogenic agents (Natori et al. 1999, Yu \& Kanost 2003, Tanji et al. 2008, Seufi et al. 2012). In our preliminary experiments, the extract of CLPT containing the CLEMs was found to be involved in suppression of $E$. coli growth (our unpublished data). In a mosquito species (Aedes aegypti) belonging to the same order (Diptera) as the flesh fly, C-type lectin was reported to be expressed in the salivary gland (Valenzuela et al. 2002). These findings indicate that CLPT is evolved in Brachyceran species after branching-off of the two sub-orders (i.e., Brachycera and Nematocera). In addition, a CLPT-like structure was observed in the blowfly (Phormia regina) in our preliminary examination (our unpublished data), which is consistent with the above assumption. These observations taken together led us to surmise that Brachyceran species acquired an organ, CLPT, in the process of evolution, in order to suppress the effect of pathogens in foods, although this remains speculation. 


\section{Acknowledgements}

We thank Dr. M. Koganezawa, Dr. I. Shimada, Dr. M. Minaba and Dr. Y. Tamada for their invaluable support and Dr. T. Hata and Mr. T. A. Inoue for their technical advice and helpful comments on histological analyses. We also thank Dr. S. Nagaoka, Dr. K. Asaoka and Dr. M. Ozaki for their stimulating advice and encouragement.

\section{References}

Bancroft, J. D. \& Gamble, M. G. (2001) Theory and Practice of Histochemical Techniques. 5th edition. Harcourt Health Sciences, New York, USA.

Denlinger, D. L. (2002) Regulation of diapause. Annu. Rev. Entomol., 47, 93-122.

Fraenkel, G. et al. (1972) Hormonal factors in cns and hemolymph of pupariating fly larvae which accelerate puparium formation and tanning. Biol. Bull., 143(1), 127-139.

Fujita, Y. et al. (1998) A novel lectin from Sarcophaga. Its purification, characterization, and cDNA cloning. J. Biol. Chem., 273(16), 9667-9672.

Hahn, D. A. \& Denlinger, D. L. (2007) Meeting the energetic demands of insect diapause: Nutrient storage and utilization. J. Insect Physiol., 53(8), 760-773.

Kawaguchi, N. et al. (1991) Involvement of Sarcophaga lectin in the development of imaginal discs of Sarcophaga peregrina in an autocrine manner. Dev. Biol., 144(1), 86-93.

Lee, R. E. et al. (1987) A rapid cold-hardening process in insects. Science, 238(4832), 1415-1417.

Natori, S. (2001) Insect lectins and innate immunity. $A d v$. Exp. Med. Biol., 484, 223-228.
Natori, S. et al. (1999) The roles of Sarcophaga defense molecules in immunity and metamorphosis. Dev. Comp. Immunol., 23(4-5), 317-328.

Oschman, J. L. \& Berridge, M. J. (1970) Structural and functional aspects of salivary fluid section in Calliphora. Tissue Cell, 2(2), 281-310.

Rinehart, J. P. et al. (2007) Up-regulation of heat shock proteins is essential for cold survival during insect diapause. Proc. Natl. Acad. Sci. U S A, 104(27), 11130-11137.

Seufi, A. M. et al. (2012) Characterization of multisugar-binding C-type lectin (SpliLec) from a bacterial-challenged cotton leafworm, Spodoptera littoralis. PLoS One, 7(8), e42795.

Takahashi, H. et al. (1985) Cloning and sequencing of cDNA of Sarcophaga peregrina humoral lectin induced on injury of the body wall. J. Biol. Chem., 260(22), 12228-12233.

Tanji, T. et al. (2008) Differential activation of the lectin and antimicrobial peptide genes in Sarcophaga peregrina (the flesh fly). Arch. Insect Biochem. Physiol., 69(4), 189-198.

Valenzuela, J. G. et al. (2002) Toward a description of the sialome of the adult female mosquito Aedes aegypti. Insect Biochem. Mol. Biol., 32(9), 1101-1122.

Verleyen, P. et al. (2004) Neuropeptidomics of the grey flesh fly, Neobellieria bullata. Biochem. Biophys. Res. Commun., 316(3), 763-770.

Yamamoto-Kihara, M. \& Kotani, E. (2004) Isolation and characterization of a C-type lectin cDNA specifically expressed in the tip of mouthparts of the flesh fly Sarcophaga peregrina. Insect Mol. Biol., 13(2), 133-140.

Yamamoto-Kihara, M. et al. (2011) Characterization of a novel C-type lectin cDNA, CLEM 20 cDNA, specifically expressed in mouthparts of the flesh fly Sarcophaga peregrina. J. Insect Biotech. Sericol., 80(1), 31-39.

Yu, X. Q. \& Kanost, M. R. (2003) Manduca sexta lipopolysaccharide-specific immulectin-2 protects larvae from bacterial infection. Dev. Comp. Immunol., 27(3), 189-196. 